\title{
Keystone meeting summary: 'Adipogenesis, obesity, and inflammation' and 'Diabetes mellitus and the control of cellular energy metabolism,' January 21-26, 2006, Vancouver, Canada
}

\author{
Silvia Corvera, ${ }^{1,6}$ Alison Burkart, ${ }^{1}$ Ja-Young Kim, ${ }^{2}$ Jennifer Christianson, ${ }^{1}$ Zhao Wang, ${ }^{2}$ \\ and Philipp E. Scherer ${ }^{2,3,4,5}$ \\ ${ }^{1}$ Program in Molecular Medicine, University of Massachusetts Medical School, Worcester, Massachusetts 01605, USA; \\ ${ }^{2}$ Department of Cell Biology, Albert Einstein College of Medicine, Bronx, New York 10461, USA; ${ }^{3}$ Department of Medicine, \\ Albert Einstein College of Medicine, Bronx, New York 10461, USA; ${ }^{4}$ Department of Diabetes Research and Training Center, \\ Albert Einstein College of Medicine, Bronx, New York 10461, USA
}

The dysregulation of specific cellular functions in adipocytes, muscle cells, $\beta$ cells, and the liver leads to changes in systemic metabolic processes and ultimately to the pathophysiological manifestations that cause type 2 diabetes. The underlying cellular mechanisms are complex. The two meetings summarized here aimed to highlight the recent advances in our understanding of the molecular basis of feeding and nutrient storage and on the molecular consequences of obesity in terms of promoting risk for type 2 diabetes and cardiovascular disease.

The worldwide epidemic in obesity has brought dramatic increases in the prevalence of chronic comorbid conditions, including insulin resistance, type 2 diabetes, the metabolic syndrome, and atherosclerosis. The rapid rise in these major public health problems underscores a growing need to understand the physiological and pathological bases of feeding and obesity, and the pathophysiological links between obesity and these associated morbidities.

At this January 21-26, 2006, gathering in Vancouver, 900 scientists from around the world gathered to exchange recent insights into the cellular deficiencies leading to the inability to properly store and use excess energy, and ultimately to insulin resistance and $\beta$-cell failure. Speakers covered most of the critical areas that are the focus of current work in many laboratories around

[Keywords: Diabetes; adipocyte; inflammation; mitochondria; ER stress] Corresponding authors.

${ }^{5}$ E-MAIL scherer@aecom.yu.edu; FAX (718) 430-8574.

${ }^{6}$ E-MAIL silvia.corvera@umassmed.edu; FAX (508) 856-1617.

Article is online at http://www.genesdev.org/cgi/doi/10.1101/gad.1447506. the world. What is the relative contribution of the brain toward insulin sensitivity in the periphery? How does inflammation fit into the picture, and what are the underlying reasons for the chronic subclinical inflammation that is observed in the context of increased fat mass? What are the upstream events that lead to increased inflammation? Does it relate to an excess of free fatty acids (FFAs)? Is it hyperglycemia that causes increased local reactive oxygen species (ROS) that trigger increased local inflammation? Is it the associated dysfunction of mitochondria? Is it a dysregulation of adipokines? Is it stress imposed on the secretory pathway under these conditions? Or is it a combination of all of these factors, each adding a piece to the yet unassembled puzzle of insulin resistance (Fig. 1)?

\section{Mitochondria and PPAR $\gamma$ coactivator-1 $\alpha$ (PGC-1 $\alpha$ )}

Obesity leads to metabolic inflexibility-i.e., an impaired switch between glucose and fat oxidation in response to homeostatic signals. Recent data from a number of laboratories highlight mitochondrial dysfunction as a key problem leading to the pathogenesis of insulin resistance associated with type 2 diabetes and aging, resulting in this metabolic inflexibility. A talk from David Kelley showed that physical activity, even in the absence of associated weight loss, causes increased mtDNA synthesis, improvements in electron transport chain activity, and higher levels of mitochondrial cardiolipin. It also increased glucose metabolism, but did not affect the activity of oxidative enzymes. Exercise and diet also lead to increased insulin sensitivity and metabolic flexibility. This indicates that there are mitochondrial adaptations 


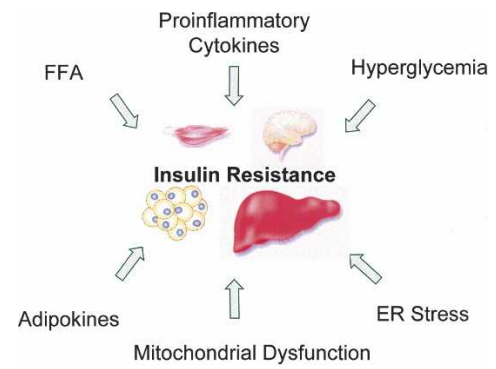

Figure 1. Major causes of insulin resistance in key target organs, such as adipose tissue, liver, muscle, and brain.

for increased fat oxidation, which may include increases in cardiolipin, NADH oxidase, and citrate synthase activities.

Mary-Elizabeth Patti reported results from gene expression analyses from human skeletal muscle, indicating that individuals with diabetes as well as those with a family history of diabetes show decreased PGC- $1 \alpha$ and PGC-1 $\beta$ as well as genes for oxidative phosphorylation in muscle biopsies. To determine whether these results are cause or effect, she studied the effects of FFAs, demonstrating that they cause a decrease in PGC-1 levels in vitro. Janet Shaw discussed basic regulatory mechanisms involved in mitochondrial function, focusing on the processes of fission and fusion. Mitochondrial networks are complex, undergoing constant restructuring. The components governing the reshaping of the mitochondrial reticulum are key to sustaining the integrity of the organellar structure. The protein Dnmlp dimerizes in the cytoplasm and is recruited to mitochondrial fission complexes (MFCs). Dnmlp then multimerizes, triggering a reorganization of Mdv1p into punctate MFCs capable of fission activity. Sheila Collins highlighted the central role of p38 mitogen-acitvated protein kinase (MAPK) in the brown adipocyte with respect to mitochondrial function. She presented data that p38 MAPK is upstream of the PGC-1-induced expression of UCP1 and that reduced levels of p38 MAPK decrease UCP1 levels. Fasting is associated with an increase in p38 activity. If this fasting-induced increase is prevented through the use of p38 small interfering RNA (siRNA), PEPCK fails to be induced, with subsequent impact on lipogenesis. This indicates that p38 may be an important molecule in the signaling cascade that induces UCP1. Michael Czech discussed the effects of another protein with surprising impact on mitochondrial gene expression. The effects of the corepressor Rip140 were examined on 3T3-L1 adipogenic gene expression and function. Depletion of Rip140 using siRNA causes an increased expression in genes involved in oxidative phosphorylation, mitochondrial biogenesis, and fatty acid oxidation pathways. Depletion of Rip140 in primary mouse white adipose tissue results in an increase in mitochondrial protein expression and oxygen consumption. The nuclear receptor ERR $\alpha$ is required for the effect of Rip140. Gerald Shulman presented his contributions toward understanding hepatic and/or muscle insulin resistance induced by FFA. He is using ${ }^{13} \mathrm{C},{ }^{31} \mathrm{P}$, and ${ }^{1} \mathrm{H}$ MRI techniques in young and old hu- man populations. Moreover, he discussed his recent findings on mitochondrial dysfunction leading to insulin resistance. Elderly patient populations show decreased function of mitochondrial oxidative-phosphorylation pathways, mitochondrial density, and mitochondrial oxidation capacity in muscle. Dale Abel presented data showing that PI3K may modulate fatty acid oxidation independent of either PGC-1 or AKT. Using mice expressing either constitutively active or dominant-negative PI3K, he showed effects on heart size and genes involved in fatty acid oxidation.

Combined, these talks highlight the important role that mitochondria play in the context of sustaining a healthy insulin-sensitive phenotype in a number of different cell types. A brief synopsis of this area can be found in Reznick and Shulman (2006).

\section{ROS and inflammation}

The PGC-1 family of transcriptional coactivators represents an important class of modulators of nuclear receptors, affecting many aspects of cellular metabolism. Bruce Spiegelman presented evidence highlighting a novel and unexpected role for the coactivator PGC- $1 \alpha$ in the transcriptional response to reactive oxygen species (ROS). Upon exposure of cells to $\mathrm{H}_{2} \mathrm{O}_{2}, \mathrm{PGC}-1 \alpha$ and PGC-1 $\beta$ are induced. In the presence of oxidative stress, a reduction of PGC- $1 \alpha$ using an $\mathrm{RNA}_{\mathrm{i}}$ approach results in a down-regulation of genes encoding proteins with anti-oxidant properties, such as UCP1, PGC- $1 \beta$, SOD-1, and SOD-2. This suggests that PGC-1 $1 \alpha$ not only plays an important role in the coordinate induction of nuclearencoded mitochondrial genes (thereby triggering increased ROS production), but is also involved in mounting a scavenging response to minimize the negative impact of these excessive ROS. PGC-1 $\alpha$ knockout mice were used to probe their susceptibility to oxidative challenges. Since these PGC-1 $1 \alpha$ knockout animals display neural dysfunction, one of the underlying reasons may be caused by enhanced levels of neuronal ROS (Lin et al. 2005).

Hyperglycemia frequently triggers an increase in ROS. Michael Brownlee further elaborated on an alternative mechanism that can increase cellular damage in diabetics as a result of increased glycolytic flux, resulting in an increase of methylglyoxal modifications on proteins. Specifically, methylglyoxal modification of mSin3A results in increased recruitment of O-GlcNAc transferase to an $\mathrm{mSin} 3 \mathrm{~A}-\mathrm{Sp} 3$ complex, with consequent increased modification of Sp3 by O-linked N-acetylglucosamine, resulting in increased angiopoietin-2 promoter activity. This offers an elegant explanation for the increased angiopoietin-2 levels observed in diabetics.

Nils-Goran Larsson presented some new data challenging the conventional model suggesting that impaired respiratory chain function in mitochondria augments ROS production and as a result increases the rate by which mtDNA mutations accumulate. This increased rate of mutations is proposed to further compromise res- 
piratory chain function. Mice expressing an error-prone version of the catalytic subunit of mtDNA polymerase accumulate a substantial burden of somatic mtDNA mutations, associated with premature aging phenotypes and reduced lifespan. Larsson therefore proposed that respiratory chain dysfunction per se is the primary inducer of premature aging. However, decreased mitochondrial matrix ROS achieved with overexpression of human SOD2 did improve mitochondrial function as judged by enhanced mitochondrial oxidative phosphorylation and tolerance.

Daniel Kelly focused on the role that ROS may play in the context of diabetic cardiac disease. Increased caloric intake increases fatty acid delivery to the heart. Kelly showed that, initially, PGC- $1 \alpha$ was able to increase mitochondrial content in response to the increase in FFA exposure. However, this compensatory mechanism fails at later stages, triggered by a drop in PGC-1 $\alpha$ levels, leading to decreased mitochondrial levels and a concomitant increase in ROS and triglyceride levels. Evan Rosen cast a wider net on the effects of reactive oxygen species in the development of insulin resistance in multiple disease states, elaborating further on the existing literature on the topic. Small molecule scavengers or the expression of catalase suppress the increase in ROS, expression of oxidative stress genes, and insulin resistance in response to TNF $\alpha$ or dexamethasone in 3T3-L1 cells. Additionally, a reduction of ROS is accompanied by a reduction of hyperglycemia in $o b / o b$ mice and an improvement of glucose tolerance without any significant effects on weight. Furthermore, SOD ${ }^{(-/+)}$mice are glucose intolerant, and humans with disorders in redox balance are insulin-resistant. Combined, this session highlighted the distinct role that ROS play in the context of insulin resistance, ultimately leading to a more generalized metabolic dysfunction affecting multiple organ systems.

\section{Inflammation and endoplasmic reticulum (ER) stress}

One of the major observations over the recent years in metabolism research relates to the close connection of insulin resistance and inflammation. Even though early observations date back as early as a decade ago (Hotamisligil and Spiegelman 1994), the full impact of inflammation has only recently been fully appreciated. Steven Shoelson discussed his recent findings on inflammation and its relationship to insulin resistance. He showed that diet-induced obesity promotes inflammation in adipose tissue and liver. Increased adiposity and hepatic steatosis increases local inflammation, leading to increased production of chemokines and cytokines. Additionally, Shoelson proposed that $\beta$-oxidation leads to ROS formation and increased NFкB activity; the established antiinflammatory effects of PPAR $\gamma$ agonists may be through transrepression of NFкB. Constitutive elevation of the inflammatory state of hepatocytes (as manifest in his LIKK mice) triggers systemic insulin resistance. Similarly, constitutive inflammation in adipocytes (as seen in FIKK mice in which constitutively active IKK $\beta$ is ex- pressed) triggers systemic insulin resistance similar to what is observed in LIKK mice. Transplantation of fat pads from these insulin-resistant mice into insulin-sensitive recipients triggers resistance in the recipient. Shoelson extended these observations by demonstrating the positive effects of salicylate treatment on insulin resistance and diabetes. Salicylate is an established inhibitor of IKK $\beta$. The observations emphasize the potential for anti-inflammatory pharmacological approaches toward improving insulin sensitivity.

Another important link to inflammation is stress within the ER. Gokhan Hotamisligil focused on the integration of stress and inflammatory pathways with metabolic homeostasis. The ER plays a critical role in these events. Obesity generates conditions that increase the demand on the secretory pathway and can lead to a constitutive induction of an ER stress response (Hotamisligil 2005). Tissues that are prominently affected by this type of stress are rich in highly active secretory cells, such as the pancreatic $\beta$ cells, the hepatocyte, and the adipocyte. Randy Kaufman presented additional data in this regard, offering a molecular link between inflammation and the ER stress response. He showed that CREBH, a new component of the ER stress response, and ATF6 synergistically activate transcription from the C-reactive protein (CRP) promoter in hepatocytes, thus linking ER stress to an acute inflammatory response (Zhang and Kaufman 2006).

Silvia Corvera presented novel data linking the ER stress response to nucleotide metabolism through increased adenylate kinase (AK) activity. AK is the enzyme catalyzing the conversion of ADP to ATP and AMP in adipose cells. She proposed that increased AK activity enhances the efficiency of ATP utilization during secretion. Myotubes depleted of AK show increased glucose uptake, oxygen consumption, and activation of AMPK, which may be a compensatory response to the decreased efficiency of ATP utilization in the absence of AK.

Phil Scherer also examined secretion and the ER stress response by focusing on adiponectin. He presented a novel mouse model with unusual fat deposition. In an $o b / o b$ background, a small increase in adiponectin levels improves all metabolic parameters generally associated with the $o b / o b$ phenotype, from insulin resistance to $\beta$-cell dysfunction. Adiponectin therefore increases lipid deposition in the adipocyte, thereby reducing lipid accumulation in other tissues and increasing insulin sensitivity. Since adiponectin is an abundant plasma protein and has a relatively short half-life, the adipocyte spends considerable resources maintaining adiponectin levels constant in circulation. This poses unique strains on the secretory pathway of the adipocyte. Adiponectin secretion is tightly controlled. A key regulator in this process is the ER chaperone ERp44, involved in the thiol-mediated retention of adiponectin. Erol-L $\alpha$ is another resident ER chaperone critically involved in mediating the interaction between ERp44 and adiponectin. Interestingly, there is a close correlation between adiponectin levels in circulation and Erol-L $\alpha$ expression in a number of different mouse models. 
A new branch of the unfolded protein response mediated by IRE-1 results in direct destabilization of mRNA coding for secretory and membrane proteins (Jonathan Weissman). These targeted transcripts contain a sequence element, which directs cleavage by the $5^{\prime}$ endonuclease Pacman. There is also a post-translational sequence element contained within the first 40 amino acids that is critical for regulation of the transcript. Therefore, under conditions of ER stress, a subset of these messages is cleaved prior to translation termination and subsequently degraded. Sonya Fonseca expanded further on the role of the WSF-1 gene in IRE-1 and Perk signaling during the ER stress response, focusing specifically on $\beta$ cells. WSF-1 appears to have an important protective role, since blocking WSF-1 function creates high levels of ER stress. The expression of WSF-1 is up-regulated in response to glucose and correlates with insulin secretion. WSF-1 also colocalizes with insulincontaining vesicles, but not with glucagon-containing vesicles.

In summary, these talks highlighted the high susceptibility of some cell types to ER stress in the context of obesity. These ER stress pathways lead to the initiation of obesity-induced inflammatory responses, and ultimately to the development of peripheral insulin resistance.

\section{Inflammatory cytokines and macrophages}

How are proinflammatory signals initiated and ultimately conveyed to other cell types? Michael Karin focused on key intracellular regulators of the IKK/NFkB pathways in the context of insulin resistance. TNF $\alpha$ and other inflammatory cytokines activate the $\mathrm{NF} \kappa \mathrm{B}$ and trigger insulin resistance. The major emphasis of his talk was on JNK1, discussing its role as a negative regulator of insulin signaling in peripheral tissues, primarily in hepatocytes through its ability to phosphorylate the insulin receptor (Karin et al. 2006). Bente Pedersen discussed another prominent inflammatory factor, IL-6, and its role in exercise. IL- 6 is produced in contracting muscles and released into circulation where it plays a role in activation/inhibition of metabolic genes, induction of lipolysis and fat oxidation, enhanced insulin sensitivity, and suppression of TNF $\alpha$. Christopher Glass discussed anti-inflammatory and antidiabetic roles of PPAR $\gamma$ in macrophages, and introduced the corepressor NCoR as a transcriptional checkpoint for a broad set of inflammatory genes in macrophages. PPAR $\gamma$ and LXR may exert their anti-inflammatory roles through decreased NCoR clearance from promoter regions of inflammatory genes (Glass and Ogawa 2006). The physiological relevance of macrophage PPAR $\gamma$ was highlighted by the finding that PPAR $\gamma$ deletion from macrophages results in insulin resistance in adipose tissue, liver, and muscle. It also resulted in a $50 \%$ reduction of efficacy of the antidiabetic class of PPAR $\gamma$ agonists, the thiazolidinediones. Lack of macrophage PPAR $\gamma$ also results in increased IKK $\beta$ levels, enhanced JNK activa- tion, and a hyperresponsiveness to LPS, consistent with an important role of PPAR $\gamma$ in macrophages as an inhibitor of inflammation. Clearly, we do not yet fully understand the full mechanism of action of PPAR $\gamma$ agonists. While critical areas of this fascinating class of antidiabetics start to emerge, we still lack a more detailed understanding of what the most critical site of action for these compounds is.

We appreciate, however, that adipose tissue is an important systemic source of inflammation. Adipose tissue contains a number of different cell types, and several recent papers demonstrate that the adipose tissue macrophage may play a very prominent role in the systemic inflammatory response. To date, we still lack a detailed understanding of why macrophages infiltrate fat tissue in the obese state, and what factors prompt these macrophages to assume a state of increased inflammation. Anthony Ferrante's observations shed some light on the role CCR2 (C-C motif chemokine receptor-2, the receptor for MCP-1) in the development of metabolic phenotypes. CCR2 plays a critical role in this process, mediating at least in part the macrophage infiltration into the growing fat pads. John Yudkin spoke on proinflammatory cytokines as the underlying complication driving the metabolic syndrome. He emphasized adipose tissue as a major source for systemic interleukin-6. This high level of IL- 6 production, along with the actions of adipose tissue-expressed $\mathrm{TNF} \alpha$ in obesity-induced insulin resistance, may underlie the associations of insulin resistance with endothelial dysfunction and coronary heart disease.

Surprising connections emerge when additional components of the innate immune system are more closely examined in the context of metabolic challenges. Jeff Flier talked about the activation of the toll-like receptor 4 (TLR-4) in the context of obesity. This pattern recognition receptor is conventionally thought of as being responsive to bacterial cell walls. However, it can also be activated by lipids. This receptor is expressed on the surface of adipocytes and is up-regulated in the obese state. Mice lacking the TLR-4 receptor fail to increase TNF $\alpha$ secretion in response to FFAs and generally display a reduced proinflammatory response. Flier also discussed some of the effects of CNTF (ciliary neurotrophic factor) that lead to a reduction in body weight in rodents and humans. He implicated neurogenesis as an important factor in the anorexic action of CNTF, and provided some data suggesting that leptin action is required to sustain CNTF action in $o b / o b$ mice.

Inflammation can be a cause and sequelae of diabetic complications. The area of diabetic complications represents a complex set of phenomena that stretch beyond inflammation proper. AnnMarie Schmidt spoke on the Receptor for Advanced Glycation Endproducts (RAGE). She proposed that in the pathogenesis of diabetic complications, the early and rapid formation of AGEs associated with hyperglycemia stimulate mechanisms that lead to recruitment of key components of the inflammatory response.

Diabetics have a dramatically increased incidence of 
cardiovascular disease. Mason Freeman spoke on atherosclerosis and the current thinking of macrophage lipid accumulation and foam cell formation. He concluded that neither the class A scavenger receptors nor CD36 are involved in the fatal conversion of macrophages to foam cells based on the observation that the loss of these proteins does not reduce arthrosclerosis. In regard to therapeutic aspects, he suggested that anti-oxidant treatment might not represent a powerful approach (Moore and Freeman 2006).

Insights into mechanisms that exert protective effects against insulin resistance sometimes come from unexpected areas of cellular physiology. Mark Febbraio spoke about the concept that heat shock proteins may convey protective effects against insulin resistance. Particularly, HSP72 seems to protect against diet-induced insulin resistance by blocking JNK phosphorylation. HSP72 overexpression resulted in lower basal glucose levels. Exposure to heat shock (thereby inducing HSP72 expression) prevents JNK activation in skeletal muscle during highfat feeding and, as a result, improves insulin sensitivity.

\section{Signaling pathways}

Impairment of the IRS/PI3-K/Akt-mediated signal transduction module is at the very core of insulin resistance phenotypes. As a result, these molecules have been under intense scrutiny for a number of years. Morris Birnbaum discussed the regulation of glucose and lipid metabolism by Akt/PKB. He summarized the phenotypes of knockout mice for the individual three Akt isoforms. While redundant in some aspects, the three isoforms play unique functions in other areas. An example is Akt2 in the context of insulin signaling in the liver. Although there is partial compensation by Akt1 for the loss of Akt2, $80 \%$ of insulin-mediated FoxO1 phosphorylation is mediated by Akt2, while only $20 \%$ is due to Akt 1 activity. Despite this difference in activity, Akt1 is able to reduce the expression of the FoxO1 target genes, PEPCK and G6Pase, in Akt2 $2^{(--)}$mice. Nevertheless, insulin is still unable to suppress glucose production in Akt2 $2^{(-1)}$ mice, and this may be due to decreased Aktmediated Ser570 phosphorylation of PGC-1 $\alpha$.

Morris White stressed the finding of largely overlapping and compensatory roles of IRS1 and IRS2 in the liver on the regulation of gluconeogenic and lipogenic gene expression. Only the loss of function of both IRS1 and IRS2 in the liver caused an increase in PEPCK, GLUT2, and PGC-1 $\alpha$ as well as major changes in glucose metabolism, a mild increase in triglyceride synthesis, and changes in whole-body insulin resistance. IRS proteins are rather big and offer a large number of regulatory phosphorylation sites. Sarah Dunn identified $>30$ Ser/ Thr phosphorylation sites on murine Irs2, but focused on Ser965, which is found phosphorylated at high levels in the basal state of serum-starved cells and slightly increased upon insulin treatment. Both basal and insulinstimulated phosphorylation events depend on the ERK/ MAP kinase cascade, and the basal phosphorylation at this site is essential for IRS2 function. Other MAPKs, such as JNK, play differential roles and are required for growth factor-induced phosphorylation. Ronald Kahn also focused on the insulin receptor signal transduction cascade. He emphasized "the critical nodes" IRS, PI3K, and AKT because all three are essential for the action of insulin, have multiple isoforms, play both positive and negative roles, and cross-talk to each other. PI3K is composed of a p110 catalytic subunit and a p85 regulatory

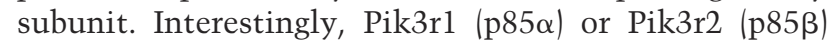
knockout mice show improved insulin sensitivity. In addition, Pik3r1-null mice display hypoglycemia, hypoinsulinemia, and decreased hepatic glucose output. Pik3r1 knockout mice decrease JNK activity and have a decreased phosphorylation state of Ser307 on IRS1 in response to insulin. The viral reconstitution of $\mathrm{p} 85$ rescues JNK activity and phosphorylation of Ser307 on IRS1.

Insulin signal transduction in the periphery has long been the only area of insulin action. However, recent exciting data on central effects of insulin highlight the important role of insulin action in the brain. Luciano Rossetti elaborated on insulin signaling in the medial basal hypothalamus $(\mathrm{MBH})$ and its impact on hepatic gluconeogenesis. Using a PI3K activator or infusing PtdIns $(3,4,5)$ P3 in the hypothalamus mimicked the effects of insulin on suppression of gluconeogenic gene expression and glucose production in the liver. These effects were inhibited in Sur1-KATP channel ${ }^{(-1)}$ mice and also in $\mathrm{Akt}^{(--) \mid}$, but not in Akt1 ${ }^{(--))}$, mice. Additionally, overfeeding rats for $3 \mathrm{~d}$ induces liver insulin resistance, which is partially alleviated with PtdIns(3,4,5)P3 infusion in the hypothalamus and completely restored with diazoxide administration. Therefore, defective brain-toliver signaling appears to be an early event in the development of insulin resistance. Other downstream targets of insulin action include the Foxo transcription factors. Domenico Accili discussed the involvement of forkhead transcription factors in diabetes and obesity, focusing on the role of the forkhead protein FoxO1 in protecting pancreatic $\beta$ cells against oxidative stress. FoxO1 forms a complex with two other proteins, Pml and Sirt1, in order to activate factors involved in transcription of the insulin gene, such as MafA. Glucose promotes FoxO1 acetylation, which targets it to PML bodies in the nucleus and protects it from ubiquitin-mediated degradation. Beyond the insulin signal transduction pathway, FoxO1 also plays a role the leptin pathway. Adenoviral overexpression of a constitutively active FoxO1 resulted in a loss of leptin action in AGRP neurons, through a mechanism that may involve competition with Stat3 for binding to AGRP and POMC promoters. FoxO1 overexpression also results in hepatic lipid accumulation, increased VLDL secretion, increased triglyceride synthesis, and decreased FFA oxidation.

AMP-activated protein kinase (AMPK) is an enzyme that serves as a fuel gauge, activated in states of low energy. AMPK can modify diverse metabolic pathways. In liver, activation of AMPK results in enhanced fatty acid oxidation and represses gluconeogenesis and production of cholesterol and triglycerides. Reuben Shaw 
described the regulation of AMPK and glucose homeostasis in the liver by the serine/threonine kinase, LKB1, and its requirement for the beneficial effects of metformin. LKB1 is required for the phosphorylation and activation of AMPK in the liver, but not in the muscle. Mice with a liver specific knockout of LKB1 have decreased P-AMPK and increased PGC- $1 \alpha$ and G6Pase expression, and they are hyperglycemic and glucose intolerant. Additionally, in the livers of these mice, the CREB coactivator TORC2 is hypophosphorylated on Ser171, leading to increased TORC2 and CREB activity and the subsequent PGC- $1 \alpha$ and gluconeogenic gene expression. TORC2 RNAi reduced PGC- $1 \alpha$ expression in the liver and blood glucose levels in the knockout mice. LKB1 therefore leads to AMPK and Sik2 activation, which then leads to the phosphorylation and nuclear exclusion of TORC2 and CREB and the reduced expression of PGC$1 \alpha$ and gluconeogenic genes. Markus Stoffel focused on insulin-regulated transcription factor FoxA2, which is phosphorylated by Akt at the critical and conserved residue Thr156. In wild-type mice, insulin phosphorylates FoxA2 and excludes it from the nucleus, resulting in an inhibition of the transcription of its target lipolytic and ketogenic genes in the liver. When a T156A FoxA2 mutant that cannot be phosphorylated is expressed, there is an increase in lipolytic, mitochondrial $\beta$-oxidation, and ketogenic genes. Adenoviral expression of this mutant in livers of $o b / o b$ mice decreased plasma glucose and insulin levels and increased oxygen consumption and heat production. Furthermore, FoxA2 appears to be required for ApoM expression and subsequent pre $\beta$-HDL formation. Therefore, decreased FoxA2 activity leads to reverse cholesterol efflux. Additional results were shown suggesting that FoxA2 phosphorylation depends on IRS1 or IRS2, while FoxO1 phosphorylation strictly depends on IRS2.

Takashi Kadowaki discussed the differences in globular and full-length adiponectin signaling: Full-length adiponectin affects the liver and muscle, signals mostly through the adiponectin receptor-2 (adipoR2), and leads to increased AMPK and PPAR $\alpha$ activity and increased fatty acid oxidation in the liver; globular adiponectin affects only muscle, signals mostly through adiponectin receptor-1 (adipoR1), and leads to p38 MAPK and AMPK activation and increased fatty acid oxidation. He discussed knockout models of adipoR1 and adipoR2 and their metabolic phenotypes, as well as presented data on adopoR1/2 double-knockout mice. These mice are viable, a finding different from data presented by Phil Scherer, who showed that double knockout mice are not viable in his hands. The differences in viability may be explained by the different knockout strategies employed, since the Kadowaki group has identified low levels of alternatively spliced messages in their double-null mice, which may explain why these mice manage to survive. Many questions remain to be answered in terms of how these receptors convey an adiponectin signal into the cytoplasm, what their specificity is, and whether or not additional receptors exist for adiponectin. Many groups have, however, described that adiponectin potently acti- vates AMPK in a number of different cell types, thereby positively affecting the cellular energy metabolism. Juleen Zierath discussed a mechanism of AMPK activation that is independent of adiponectin; AMPK can be activated pharmacologically by 5 -aminoimidazole-4-carboxamide-1- $\beta$-4-ribofuranoside (AICAR), which is phosphorylated in vivo to the AMP analog AICAR-monophosphate (ZMP). Treatment with AICAR leads to a striking normalization of blood glucose levels and glucose tolerance in $o b / o b$ mice after in vivo treatment, providing evidence that targeting the AMPK pathway effectively improves metabolic defects in diabetes.

\section{From genes to insulin resistance}

The genetics of type 2 diabetes are unquestionably very heterogeneous. However, an increasing number of single point mutations are being identified that lead to profound phenotypic changes at the level of metabolism. Steven O'Rahilly discussed some recent insights from his laboratory. The mutations discussed include a missense mutation in Akt2 (R274H), mutations in the ligand or DNA-binding domain of PPAR $\gamma$, and mutations in the leptin receptor and proopiomelanocortin (POMC). He also discussed the relationship between the PPAR $\gamma$ ligand-resistance syndrome (PLR) and metabolic disorders. PLR syndrome patients show insulin resistance, dyslipidemia, hypertension, and partial lipodystrophy, which highlights the importance of the physiological functions of PPAR $\gamma$. However, PPAR $\gamma$ P467L knock-in mice show hypertension, but do not develop insulin resistance even after a high-fat diet. The P467L PPAR $\gamma$ mutation expressed on an $o b / o b$ background results in impaired adipocyte function and expandability. Lastly, he presented the findings of a new human leptin receptor mutation located at a splicing donor site. This mutation is more common than other leptin mutations, but does not lead to a change in plasma leptin levels.

\section{Adipogenesis and energy metabolism}

A number of talks focused on the cellular physiology of the adipocyte. One of the hallmark features of the adipocyte is its ability to store large amounts of lipids. Lipid droplet formation, stabilization, and degradation are governed by a number of lipid droplet-binding proteins. Perry Bickel provided an overview of the "PAT" family of lipid droplet-binding proteins, whose members are perilipins, adipophilin, Tip47, Oxpat, and S3-12. During the transition from a nascent lipid droplet toward a mature lipid droplet, there is a gradual interchange of these binding proteins on the surface of these droplets. The lipid droplets emerge at the site of triglyceride synthesis in the ER and immediately associate with the PAT family members (Londos et al. 2005).

Stephen Farmer's talk covered the important area of transcriptional regulation governing the process of adipogenesis. He described the relationship between $\mathrm{C} / \mathrm{EBP} \beta$ and PPAR $\gamma$. PPAR $\gamma$ activates $\mathrm{C} / \mathrm{EBP} \alpha$ expres- 
sion during adipogenesis. $\mathrm{C} / \mathrm{EBP} \alpha$ plays a critical role in the regulation of adipogenic gene expression, especially at later stages of the differentiation process. Activation of PPAR $\gamma$ by C/EBP $\beta$ induces a decrease in binding of HDAC1 to the C/EBP $\alpha$ promoter by enhancing the degradation of HDAC1. To further determine the mechanism of PPAR $\gamma$ activation by C/EBP $\beta$, he suggested that the phosphorylation of C/EBP $\beta$ at Thr188 is critically involved and achieved through the MEK/ERK pathway. $\mathrm{He}$ also reported that $\mathrm{C} / \mathrm{EBP} \alpha$ is the essential molecule regulating the expression of adiponectin. Unlike other adipocyte-specific proteins such as FAS and aP2, which need only PPAR $\gamma$ for their expression, adiponectin expression requires both PPAR $\gamma$ and C/EBP $\alpha$ (Farmer 2005). Dan Lane continued this topic by showing evidence for post-translational modification of C/EBP $\beta$ by MAP kinase and GSK $\beta$ during mitotic clonal expansion (MCE) and adipogenesis. The sequential phosphorylation of $\mathrm{C} / \mathrm{EBP} \beta$ is required for the acquisition of DNA-binding activity of C/EBP $\beta$ to activate expression of C/EBP $\beta$ and PPAR $\gamma$. When the differentiation is initiated in 3T3L1 cells, Thr 188 residue of C/EBP $\beta$ is phosphorylated by MAPK at a very early time point, followed by Ser184/ Thr179 phosphorylation by GSK $\beta \sim 14$ h later. He suggested that this long pause correlates with GSK $\beta$ translocation to the nucleus and cellular entry into $S$ phase to promote $\mathrm{MCE}$, which is required for terminal differentiation. Additionally, inhibitors of MAP kinase or GSK $\beta$ block both MCE and adipocytes differentiation. Ormond MacDougald discussed the function of Wnt10b on commitment steps within the mesenchymal lineage. Wnt10b inhibits differentiation of preadipocytes in transgenic mice. These mice are lean because of decreased fat mass, and resistant to diet-induced obesity with improved insulin sensitivity. Moreover, these mice show an increased bone density compared with wildtype mice. They are also resistant to bone loss induced by estrogen depletion or aging. In line with these results, Wnt10b-null mice have decreased bone mass in the distal femur. He concluded that Wnt10b promotes osteoclast differentiation of mesenchymal cells by activating the osteoblastogenic pathway and blocking the adipogenic pathway. Shigeki Shimba described the involvement of BMAL1 in adipogenesis, a transcription factor that regulates circadian rhythm. BMAL1 is expressed abundantly in the brain and adipose tissue and its expression is up-regulated during adipocyte differentiation or by high-fat feeding. He proposed a metabolic loop mediated by BMAL1, which regulates lipogenesis by increased expression of SREBP-1 and Rev-erbA activity. Therefore, FFAs induce BMAL1 expression, which leads to glucose uptake, cholesterol synthesis, and lipid accumulation and inhibits $\beta$-oxidation and lipolysis.

\section{Novel molecules and metabolic pathways}

Rudolf Zechner provided important new evidence that adipocyte triglyceride lipase (ATGL) exerts a rate-limiting role in lipolysis, a finding that explains the lack of a lipolytic phenotype in mice lacking hormone-sensitive lipase. Results shown included in vitro studies in 3T3-L1 adipocytes where overexpression of ATGL resulted in increased triglyceride mobilization. Furthermore, ATGL knockout mice have increased adipose tissue mass, most evident in brown adipose tissue, due to the decreased lipolytic activity in all fat depots. The ATGL-deficient mice also display increased triglyceride accumulation in many tissues, particularly heart and muscle, yet display improved glucose tolerance and insulin sensitivity.

Barbara Kahn discussed a novel and exciting new adipokine, retinol-binding protein 4 (RBP-4), and its impact on the metabolic syndrome. The serum levels of RBP-4 are elevated in rodent models of insulin resistance as well as obese nondiabetic, glucose-intolerant, and type 2 diabetic patients. There is also a negative correlation of RBP-4 with glucose disposal and GLUT4 levels in nonobese humans with a family history of insulin resistance, and therefore RBP-4 may serve as a marker for high-risk patients. Tissue expression of RBP-4 is highest in the liver and adipose tissue, but expression increases only in the adipose tissue in insulin-resistant states. Furthermore, RBP-4 seems to cause metabolic abnormalities, because PEPCK expression increases in the liver of wild-type mice treated for $2 \mathrm{wk}$ with purified RBP-4, and these mice also develop insulin resistance and glucose intolerance. Lastly, she discussed the benefits of fenretinide (an RBP-4 inhibitor) treatment in mice fed a highfat diet; fenretinide prevented glucose intolerance and insulin resistance in the liver and muscle and improved glucose production in the liver.

Chris Newgard used the Keynote Address to present an overview of new tools for use in metabolic profiling ("metabolomics"), including the use of GC/MS, MS/MS, and LC/MS, and NMR-based analysis of isotopes along with bioinformatics and modeling tools to measure metabolic flux. He presented three examples with applied metabolomics: (1) the mechanism of glucosestimulated insulin secretion and its impairment in type 2 diabetes; (2) the mechanism of high-fat, diet-induced insulin resistance; and (3) a translational component, focusing on the metabolic profiling during weight loss in human subjects. In the first example, pyruvate cycling, through the pyruvate isocitrate shuttle, was shown to be critical in regulating the glucose response in mitochondria. In the second example, Newgard demonstrated that expression of malonyl-CoA decarboxylase in the liver of mice reversed liver, muscle, and whole-animal insulin resistance. In addition, a high-fat diet induced serum levels of hydroxybutyrylcarnitine ketones and promoted muscle ketone dysregulation. In the third example, metabolic weight-loss markers, both independent of the intervention and dependent of the intervention, were defined. Together, through the use of metabolomics, biochemistry and cell signaling can be related to metabolic intermediates and provide insights into mechanisms that underlie obesity and diabetes.

Richard N. Bergman highlighted recent insights in the pathogenesis of the metabolic syndrome. He showed that hepatic insulin resistance is linked to increased vis- 
ceral adiposity. As visceral adiposity increases in dogs, an increase in insulin resistance is observed along with an increase in expression of genes involved in lipolysis within the visceral depots as well as an increase in gluconeogenic genes in liver. Adipokine expression (IL-6, $\mathrm{TNF} \alpha$, resistin, and adiponectin) at the mRNA level did not change under these conditions, but no information on protein levels was available. Blood glucose and FFAs fluctuate throughout the day in order to maintain adequate levels of fuel. He reported that an increased pulsatile release of FFAs, occurring predominantly at night, seem to induce hepatic insulin resistance more than increased FFA levels alone. This study supports the "portal theory," which suggests that hepatic insulin resistance is a result of an increase in FFA flux from the visceral fat depot. Hyperinsulinemic compensation is the result of a $60 \%$ increase in FFA at night, stimulating enhanced insulin release from $\beta$ cells.

Mitch Lazar first highlighted the role of resistin in insulin resistance and atherosclerosis in mice and humans. The levels of resistin are elevated in obese states, and hyper-resistinemia promotes insulin resistance. He also discussed the down-regulation of the negative component of the circadian clock Rev-erb $\alpha$, which must be phosphorylated by GSK3 $\beta$ to maintain stability and activity.

\section{Therapeutic targets}

Peter Cornelius showed some additional data on the CCR2 KO mice and the effect of diet-induced obesity. CCR2 KO mice on a high-fat diet gain little weight, are more insulin-sensitive, and tend to have smaller adipocytes, suggesting that the MCP-1 pathway may be an a worthwhile pharmacological target. Bei B. Zhang discussed the role of DPP-IV inhibitors in glycemic control and $\beta$-cell preservation. Chronic DPP-IV inhibitor treatment in streptozotocin-diabetic mice leads to improved islet morphology, increased $\beta$-cell mass, increased insulin secretion, and decreased $\alpha$-cell mass. Thus, DPP-IV inhibitors may be useful in the treatment of type 2 diabetes.

\section{Concluding remarks}

While we realize that we have great difficulty providing an exact numerical value to define "obesity" and "overweight," we can resort to a more functional definition by stating that "obesity" is a degree of adiposity that causes impairment in normal motor or metabolic function, including but not limited to the metabolic consequences that have been characterized in many of the presentations heard at this meeting. We could define it as a state that includes (but is not limited to) a deregulation of adipokines and other products of adipocytes, a deregulation of inflammation in adipose tissue and other organs, involving the recruitment of macrophages and other immune cells, that ultimately leads to an accumulation of lipids in nonadipose tissue. This results in mitochon- drial dysfunction and insulin resistance at the level of key organs, such as the liver, muscle, and the heart, with profound effects on the vasculature. Combined, this describes a set of symptoms that we could functionally define as the metabolic syndrome and associated atherosclerosis. We are going through a very exciting time in metabolism, in part because the field has attracted researchers and their enabling technologies from a variety of specialties that have traditionally not devoted much effort to the problem of metabolic deregulation. The purpose of meetings such as the two described here is to bring the different subspecialties together, along with findings from both the basic and clinical environment, so we can learn from each other and highlight what the critical questions are for the future. Perhaps the biggest challenge at this stage is to provide an integrative model of insulin resistance that can unify the exciting (and occasionally diametrically opposed) hypotheses presented at this meeting. Which phenomena are causes and which are effects of insulin resistance? What are the earliest clinical manifestations of insulin resistance? Which cell types are primarily affected at these very early stages, and which mechanisms lead to such sensitivities? Once affected, how do these cell types contribute and further exacerbate the insulin resistance syndrome? Does the adipocyte play a more prominent role in this process than other cell types, and if yes, what aspects of adipocyte function are most significant in this context (Scherer 2006)? We look forward to hearing (at least partial) answers at the next Keystone meeting devoted to the topic in 2007.

\section{Acknowledgments}

This meeting was supported by NIH grants R13-DK072700 and R13-DK074300. We thank Tayeba Khan, Deborah Brancho, and Todd Schraw for additional contributions to this report, and acknowledge the contributions made by the co-organizers of these meetings, Steven Shoelson, Bruce Spiegelman, and Luciano Rossetti, as well as the Keystone Symposium Organization for their logistical support. Our apologies to the speakers whose talks were not specifically mentioned due to space restriction.

\section{References}

Farmer, S.R. 2005. Regulation of PPAR $\gamma$ activity during adipogenesis. Int. J. Obes. (Lond.) (Suppl. 1) 29: S13-S16.

Glass, C.K. and Ogawa, S. 2006. Combinatorial roles of nuclear receptors in inflammation and immunity. Nat. Rev. Immunol. 6: 44-55.

Hotamisligil, G.S. 2005. Role of endoplasmic reticulum stress and c-Jun NH2-terminal kinase pathways in inflammation and origin of obesity and diabetes. Diabetes (Suppl. 2) 54: S73-S78.

Hotamisligil, G.S. and Spiegelman, B.M. 1994. Tumor necrosis factor $\alpha$ : A key component of the obesity-diabetes link. Diabetes 43: 1271-1278.

Karin, M., Lawrence, T., and Nizet, V. 2006. Innate immunity gone awry: Linking microbial infections to chronic inflammation and cancer. Cell 124: 823-835. 
Lin, J., Handschin, C., and Spiegelman, B.M. 2005. Metabolic control through the PGC-1 family of transcription coactivators. Cell Metab. 1: 361-370.

Londos, C., Sztalryd, C., Tansey, J.T., and Kimmel, A.R. 2005. Role of PAT proteins in lipid metabolism. Biochimie 87: 45-49.

Moore, K.J. and Freeman, M.W. 2006. Scavenger receptors in atherosclerosis. Beyond lipid uptake. Arterioscler. Thromb. Vasc. Biol. (in press).

Reznick, R.M. and Shulman, G.I. 2006. The role of AMP-activated protein kinase in mitochondrial biogenesis. J. Physiol. 574: 33-39.

Scherer, P.E. 2006. Adipose tissue: From lipid storage compartment to endocrine organ. Diabetes 55: 1537-1545.

Zhang, K. and Kaufman, R.J. 2006. Protein folding in the endoplasmic reticulum and the unfolded protein response. Handb. Exp. Pharmacol. 172: 69-91. 


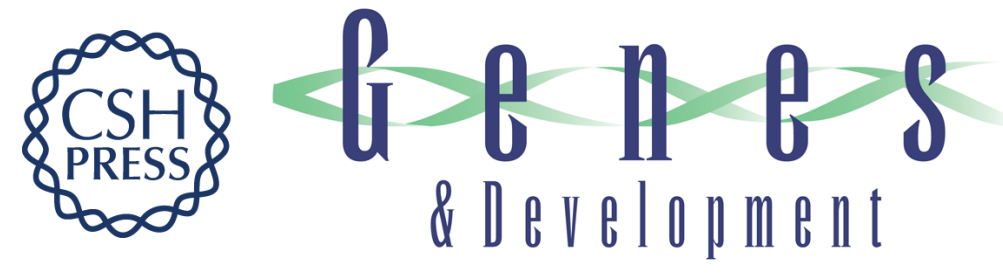

\section{Keystone meeting summary: 'Adipogenesis, obesity, and inflammation' and 'Diabetes mellitus and the control of cellular energy metabolism, ' January 21 -26, 2006, Vancouver, Canada}

Silvia Corvera, Alison Burkart, Ja-Young Kim, et al.

Genes Dev. 2006, 20:

Access the most recent version at doi:10.1101/gad.1447506

References This article cites 9 articles, 2 of which can be accessed free at:

http://genesdev.cshlp.org/content/20/16/2193.full.html\#ref-list-1

License

Email Alerting Receive free email alerts when new articles cite this article - sign up in the box at the top

Service right corner of the article or click here.

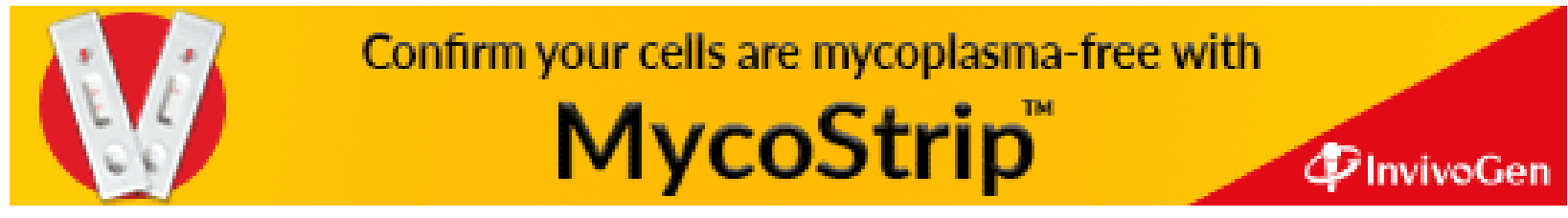

